\title{
Incidência de opacificação de cápsula posterior em pacientes submetidos à facoemulsificação e implante de lentes intra-oculares acrílicas hidrofílicas expansíveis
}

\author{
Incidence of posterior capsule opacification in patients submitted to \\ phacoemulsification and expandable acrylic intraocularlens implantation
}

\author{
Hilton Arcoverde Gonçalves de Medeiros $^{1}$ \\ Marcos Ávila² \\ Procópio Miguel dos Santos ${ }^{3}$
}

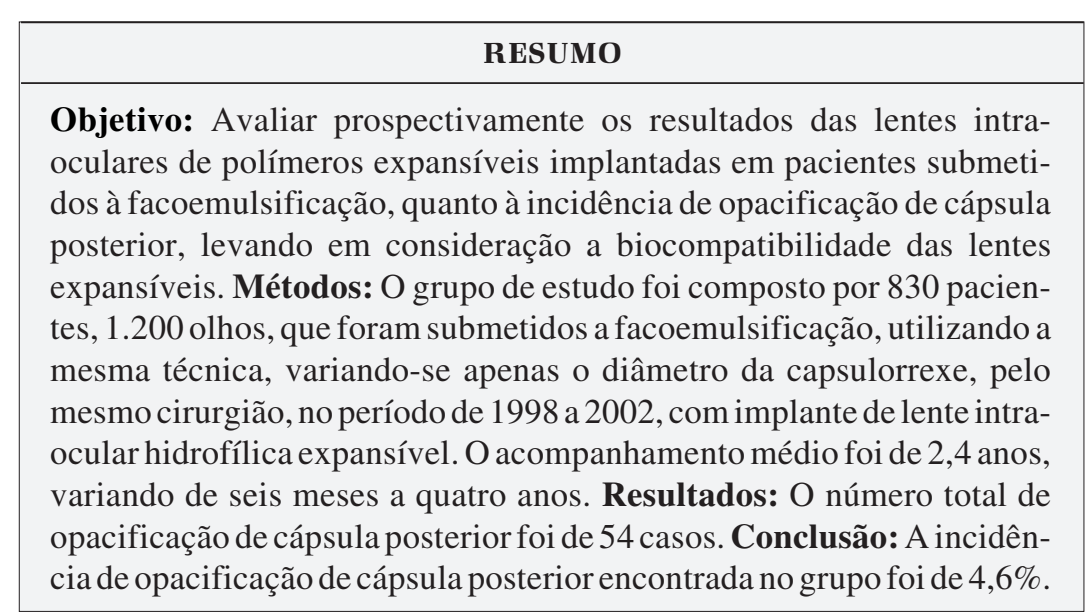

Descritores: Implante de lente intra-ocular; Lentes de contato hidrofílicas; Cápsula do cristalino; Acuidade visual; Facoemulsificação; Capsulorrexe

\section{INTRODUÇÃ̃O}

Com o advento da facoemulsificação e o implante de lentes intra-oculares dobráveis (LIO), uma nova etapa foi introduzida na oftalmologia. A era da cirurgia sem sutura. Para isso, existe a necessidade de um implante de LIO através de incisões auto-selantes. As lentes dobráveis, que são implantadas através de pequena incisão, tornaram-se amplamente difundidas, devido à rápida recuperação funcional e menor incidência de complicações (astigmatismo, opacificação da cápsula posterior, descentralização provocada pela síndrome da fibrose capsular), quando comparadas com as LIO rígidas ${ }^{(1)}$.

Essas complicações foram minimizadas, com o desenvolvimento das lentes acrílicas dobráveis hidrofílicas. Entretanto, novas complicações foram relatadas, principalmente a descentração e a opacificação da óptica da lente ${ }^{(2)}$.

As lentes dobráveis utilizadas atualmente podem ser lentes de peça única, ou seja, as alças são do mesmo material do corpo óptico da lente, e fundidas em uma peça de acrílico hidrofóbico. As bordas são quadradas, o que cria uma barreira física impedindo a migração de células do equador capsular para o pólo posterior da cápsula posterior, diminuindo sensivelmente a incidência de opacificação de cápsula posterior. Um exemplo desta geração de lentes são as Acrysof ${ }^{\circledR}$ (Alcon, 6201 South Freeway, Fort Worth, TX, USA) modelo SA30AT, que podem ser inseridas por incisões 
372 Incidência de opacificação de cápsula posterior em pacientes submetidos à facoemulsificação e implante de lentes intra-oculares acrílicas hidrofílicas expansíveis

de 2,75 mm a 3,2 mm. Possuindo uma excelente biocompatibilidade capsular, é hoje uma das melhores alternativas, levando a resultados bastante satisfatórios, sendo, entretanto elevado o seu custo, o que a torna inacessível para grande parte da população $^{(3)}$.

Apareceram, então, no mercado, as lentes expansíveis, com bom índice de biocompatibilidade, onde a maior parte do conteúdo da lente expandida no meio intra-ocular é o próprio humor aquoso. O modelo mais difundido deste tipo de lente é a hidrofílica expansível de hidrogel ${ }^{(1)}$. Este produto está comercialmente disponível sob o nome Acqua ${ }^{\circledR}$ (Mediphacos, Belo Horizonte, Minas Gerais, Brasil).

São implantadas em seu estado desidratado por uma incisão de 3,2 mm, não necessitando de injetor ou mecanismo especial para dobrar, graças ao seu tamanho, que, neste estado, é de 3,2 mm de diâmetro por 5,7 $\mathrm{mm}$ de comprimento. Estas lentes, em prato, são anguladas posteriormente $15^{\circ}$, possuindo dois orifícios de fixação em suas extremidades, com a finalidade de permitir uma fibrose inter-capsular, responsável pela estabilização da lente ${ }^{(4)}$.

O objetivo deste estudo foi avaliar prospectivamente os resultados das LIOs de polímeros expansíveis implantadas em pacientes submetidos à facoemulsificação, quanto ao percentual de opacificação de cápsula posterior, levando em consideração a biocompatibilidade das lentes expansíveis.

\section{MÉTODOS}

A população amostral foi composta de 830 pacientes (1.200 olhos) submetidos à facoemulsificação no período de 1998 a 2002, onde nenhum paciente possuía outra causa diagnosticável de baixa de acuidade visual, que não a catarata. Foram excluídos do estudo, pacientes diabéticos, com glaucoma e com patologias retinianas. No primeiro ano foram submetidos à cirurgia, 285 olhos, seguidos de 308 olhos no ano de 1999, 293 olhos no ano de 2000, 314 no ano de 2001. Todos os olhos receberam LIO hidrofílicas expansíveis Acqua ${ }^{\circledR}$ (Mediphacos, Brasil) (Figuras 1 a 3).

O procedimento cirúrgico consistiu de uma incisão em córnea clara de 3,2 mm, localizada às $10 \mathrm{~h}$, em todos os olhos, seguido pela realização de capsulorrexe contínua e circular de 5 mm nos anos de 1998 e 1999, tendo sido ampliada para $6 \mathrm{~mm}$, procedendo-se a facoemulsificação nuclear utilizandose solução de Ringer com Lactato e implante da lente intraocular, procurando mantê-las na posição vertical, ou no máximo oblíqua, evitando sempre a posição horizontal. As figuras 4 e 5 demonstram o procedimento de inserção e posicionamento da lente no pós-operatório imediato.

As LIOs expansíveis são inseridas em seu estado desidratado, permitindo seu implante através de incisão de $3,2 \mathrm{~mm}$. O procedimento padrão foi uma capsulorrexe de 5 a $6 \mathrm{~mm}$, com a câmara anterior preenchida com visco-elástico tipo metilcelulose, procedendo-se a emulsificação do material nuclear, seguida da implantação da lente intra-ocular, com a câmara ante- rior preenchida por visco-elástico tipo hialuronato de sódio com alto peso molecular, mantendo o saco capsular preenchido durante todo o processo de implantação da lente ${ }^{(5)}$.

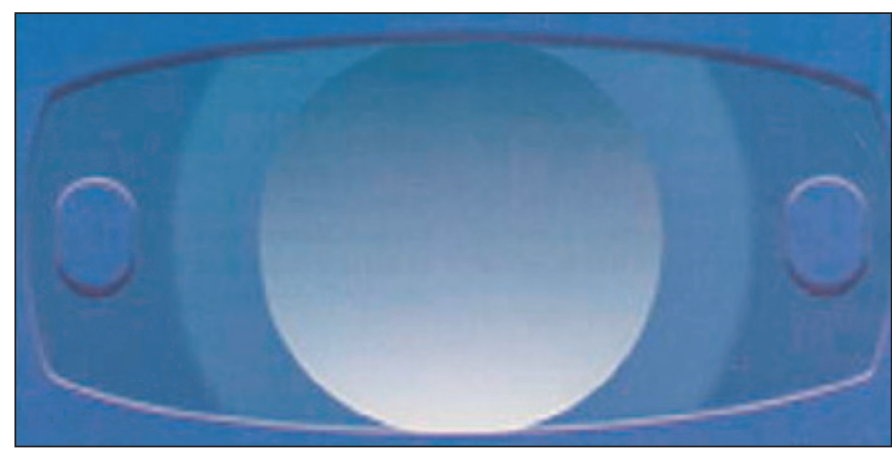

Figura 1 - Vista frontal da lente desidratada (Fonte: Mediphacos, Brasil)

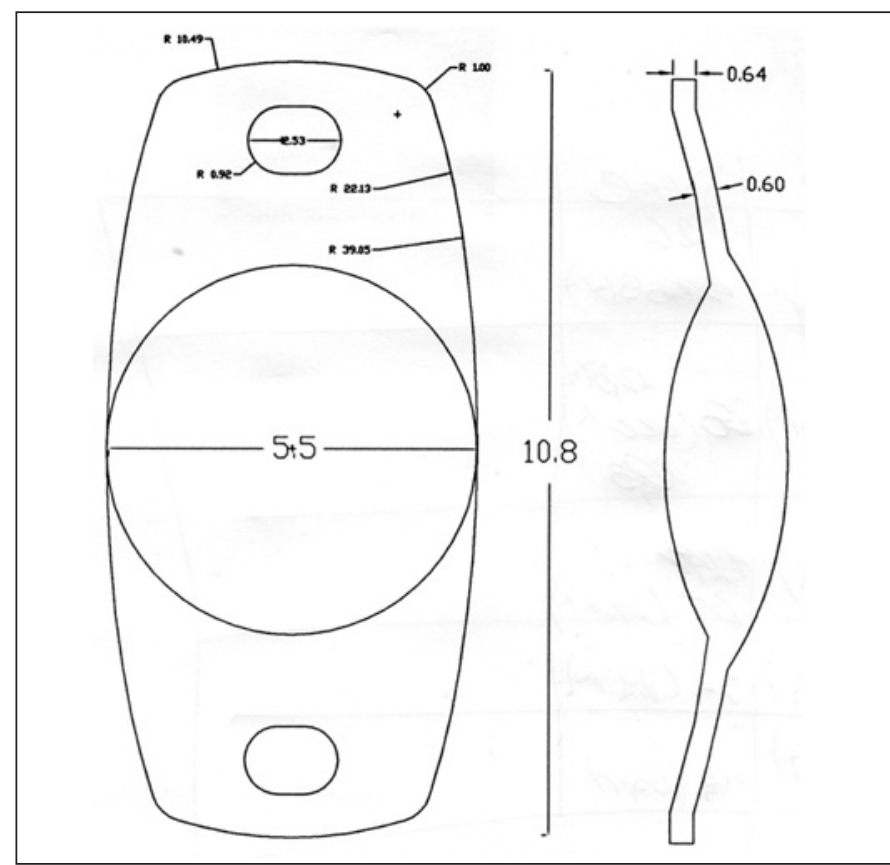

Figura 2 - Desenho da lente após expansão (Fonte: Mediphacos, Brasil)

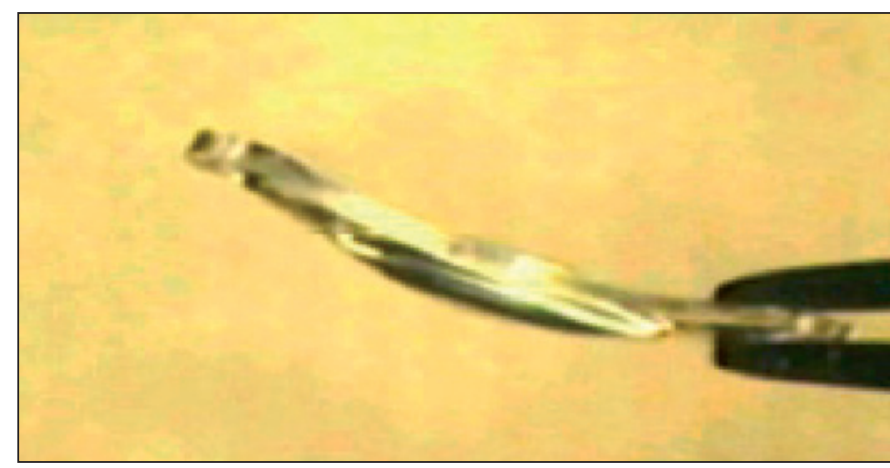

Figura 3 - Aspecto em perfil mostrando angulação (Fonte: Mediphacos, Brasil) 
Todos os pacientes foram submetidos a uma revisão pósoperatória sistematizada realizada pelo mesmo oftalmologista no primeiro dia pós-operatório, e, após, nos dias 5, 15, 30, e, aos 12 meses, por outro especialista em segmento anterior. Com 12 meses aqueles que não compareceram foram descartados do estudo, de tal modo que a população amostral é composta somente dos pacientes que se apresentaram.

Em todas as revisões foram avaliadas a acuidade visual sem correção (AV), a acuidade visual melhor corrigida (AVMC), a refração residual, a existência de opacificação da cápsula posterior (OCP), a medição da pressão intra-ocular com tonometria de aplanação, e realizada oftalmoscopia binocular indireta.

Para análise dos dados, foram considerados que o paciente com mais de 6 meses de completado aniversário de cirurgia foi incluído no grupo seguinte, ou seja, paciente com 1 ano e 5 meses foi considerado com 1 ano e paciente com 1 ano e 7 meses foi considerado com 2 anos.

As opacificações capsulares foram avaliadas, subjetivamente, através da retroiluminação ${ }^{(6)}$ e segundo sua intensidade,

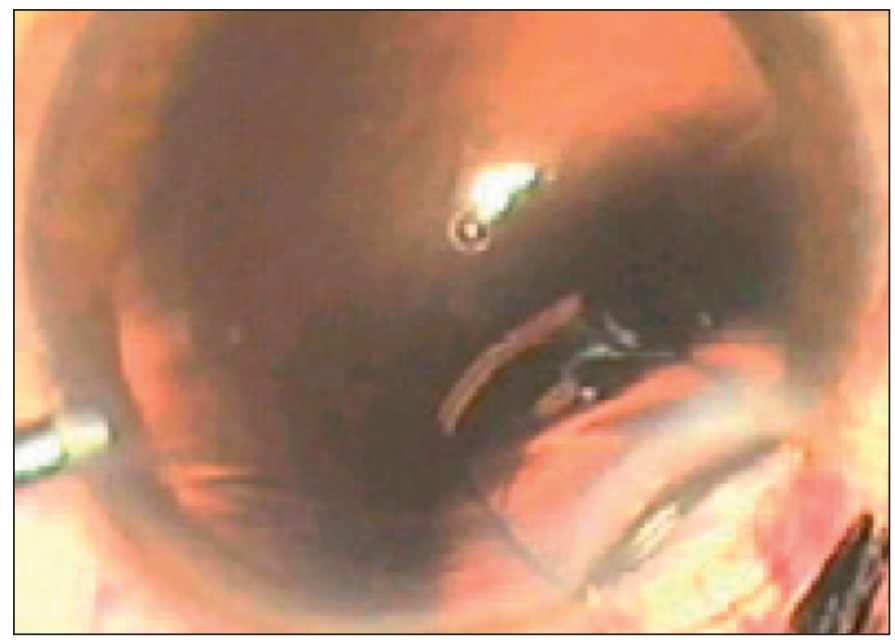

Figura 4 - Inserção da lente (Fonte: Foto dos autores)

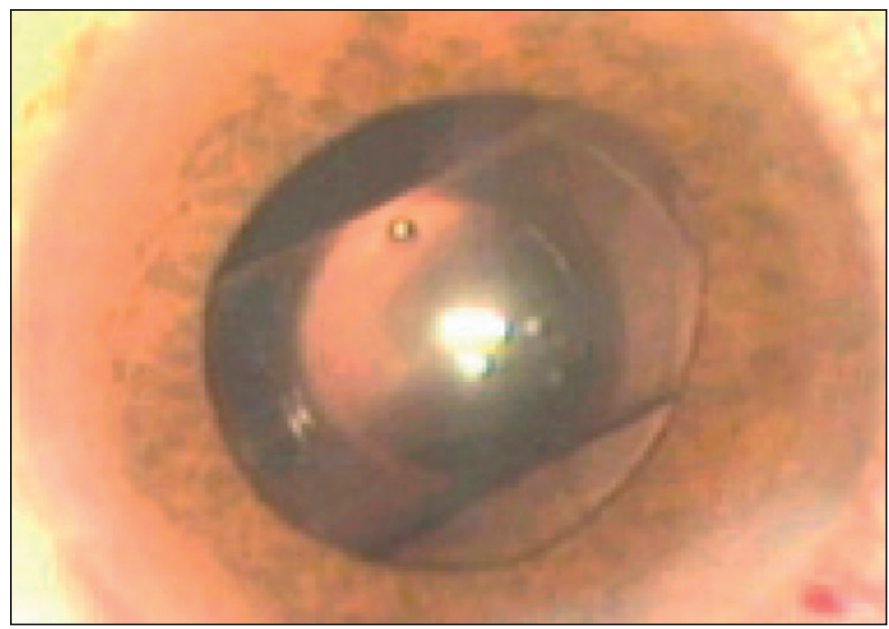

Figura 5 - Aspecto do pós-operatório imediato (Fonte: Foto dos autores) sendo atribuída uma escala crescente, de tal forma que se havia opacificação leve, sem interferência na $\mathrm{AV}$, era classificada como $1+$, se afetasse a AV, até 20/30,2+, e com AV inferior a 20/30, 3+. Nenhum paciente possuía outras causas prévias de baixa de AV, de acordo com os critérios de exclusão.

Foi observada ainda, a incidência de descentração da LIO, se houvesse. Neste último caso foi considerado $1+$, para descentrações de até $1 \mathrm{~mm}$ do centro pupilar; $2+$, descentrações de 1 a 2 mm do centro pupilar; e, $3+$ descentrações acima de $3 \mathrm{~mm}$ do centro da pupila.

Foram realizadas análises de estatística descritiva e de inferência estatística. A primeira analisa os resultados obtidos na amostra, a segunda extrapola e tira conclusões a respeito da população ${ }^{(7)}$. Para a análise descritiva dos dados utilizamos a representação tabular e gráfica. Já para a inferência estatística foram elaborados intervalos de confiança e testes de hipóteses. Com isso a distribuição dos resultados pode ser aproximada à distribuição normal padrão( ${ }^{(8)}$.

Os intervalos de confiança tiveram $95 \%$ de confiabilidade.

Foi utilizado o Teste Qui-Quadrado para provar algumas hipóteses de igualdade de proporção e média. Para isso foi calculado o p-valor do teste. Definiu-se como verdadeira a hipótese a ser provada se o p-valor for menor que $0,05^{(9)}$.

\section{RESULTADOS}

Do total das 1.500 lentes implantadas, 169 olhos tiveram apenas 6 meses de seguimento, e, 131 olhos não compareceram aos controles, sendo, então, descartados do protocolo.

Os 1.200 restantes estão assim distribuídos: $285 \mathrm{com}$ seguimento de 4 anos, 308 com 3 anos, 293 com 2 anos e 314 com 1 ano. A tabela 1 mostra a evolução das complicações por grupo. O grupo 1 corresponde aos olhos operados em 1998 (285 olhos de 215 pacientes); o grupo 2, aloca os operados em 1999 (308 olhos de 176 pacientes); no grupo 3, se encontram os operados em 2000 (293 olhos de 189 pacientes) e o grupo 4 contém os operados em 2001 (314 olhos de 174 pacientes). A idade média foi de 66,8 anos, variando de 51 a 84 anos, com $58,3 \%$ dos pacientes sendo do sexo feminino.

Ao total, o número de opacificações de cápsulas posteriores foi de 54 casos $(4,6 \%)$ (Tabela 2). Os pacientes identificados com OCP 2+ ou 3+, eram submetidos a capsulotomia com YAG-laser e excluídos dos controles posteriores.

\begin{tabular}{|lcc|}
\hline \multicolumn{3}{|l|}{$\begin{array}{l}\text { Tabela 1. Pacientes submetidos à facoemulsificação com implante } \\
\text { de lentes intra-oculares acrílicas hidrofílicas, com a freqüência de } \\
\text { OCP, separadas pelos grupos }\end{array}$} \\
Grupo & Ano & OCP (2+ ou 3+) \\
Grupo 1 & 1998 & 13 olhos \\
Grupo 2 & 1999 & 16 olhos \\
Grupo 3 & 2000 & 14 olhos \\
Grupo 4 & 2001 & 11 olhos \\
OCP= opacificação de cápsula posterior & \\
\hline
\end{tabular}


374 Incidência de opacificação de cápsula posterior em pacientes submetidos à facoemulsificação e implante de

lentes intra-oculares acrílicas hidrofílicas expansíveis

\begin{tabular}{|c|c|c|c|}
\hline Complicações/ano & $\begin{array}{l}\text { Olhos } \\
\text { operados }\end{array}$ & $\begin{array}{c}\text { OCP } \\
\text { encontradas }\end{array}$ & Percentual \\
\hline $1^{\circ}$ ano $\mathrm{OCP}$ & 285 & 13 & $4,5 \%$ \\
\hline $2^{\circ}$ ano OCP & 308 & 16 & $5,1 \%$ \\
\hline $3^{\circ}$ ano OCP & 293 & 14 & $4,7 \%$ \\
\hline $4^{\circ}$ ano $\mathrm{OCP}$ & 314 & 11 & $3,5 \%$ \\
\hline Total OCP & 1200 & 54 & $4,6 \%$ \\
\hline
\end{tabular}

Nos anos de 1998 e 1999, era realizada uma capsulorrexe de $5 \mathrm{~mm}$, a qual foi ampliada para $6 \mathrm{~mm}$, a partir de 2000, face ao aparecimento de 38 casos de fimose da cápsula anterior e da incidência de descentração acima de $1 \mathrm{~mm}$ da LIO, o que foi encontrado em 49 pacientes $(7,46 \%)$, não tendo sido encontrada nenhuma descentração acima de $2 \mathrm{~mm}$. Por ocasião da última revisão, foram encontrados 38 casos de opacificação de cápsula anterior, no total dos grupos $(2,53 \%)$ e 205 olhos apresentaram formação de anel de Soemmering $(13,7 \%)$. Não se encontrou diferença estatisticamente significativa quanto à incidência de OCP nos pacientes submetidos à capsulorrexe de $5 \mathrm{~mm}$ quando comparados aos pacientes submetidos à capsulorrexe de $6 \mathrm{~mm}$.

\section{DISCUSSÃO}

Devido ao fato de ser altamente hidrofílica, uma vez implantada, a lente inicia um rápido processo de expansão, interagindo com o visco elástico, com a solução salina e com o humor aquoso, atingindo aproximadamente $90 \%$ de seu tamanho final com 15 minutos; porém aos 2 minutos o crescimento já é suficiente para impedir um deslocamento para fora do saco capsular. O tamanho final da lente expandida ou hidratada é de 5,2 mm de diâmetro e de 10,8 mm de comprimento (Medipha$\cos$, Brasil) $)^{(4)}$.

As LIO expansíveis de hidrogel são confeccionadas com o polímero “Acryfil CQ". Um polímero altamente hidratado feito a partir do pHEMA (poli hidroximetilmetacrilato), vinil pirolidona e metil-metacrilato com adição de componente capaz de absorver ultra-violetas A e B.

O desenho (utilizando o conceito de "square edge", ou bordas retas) e o material da LIO acrílica hidrofílica expansível ajudam a diminuir a incidência de opacificação de cápsula posterior. Hollick ${ }^{(10)}$ encontra uma maior incidência de OCP em olhos com LIO de hidrogel, porém vale salientar que as LIO utilizadas não possuíam as bordas retas, que para diversos autores $^{(10-13)}$ é o principal fator envolvido na prevenção da OCP. Quando necessário, devido ao alto teor de água em sua composição, as marcas produzidas em decorrência da aplicação de YAG-Laser na superfície posterior da LIO hidrofílica expansível, são praticamente imperceptíveis após $24 \mathrm{~h}^{(4)}$.

Como todo procedimento cirúrgico, certas complicações são possíveis. As principais complicações relacionadas ao implante de lentes intra-oculares pós-extração da catarata são a opacidade de cápsula posterior, descentração, luxação anterior ou posterior, membrana pupilar e fimose da LIO, inflamação, pressão intra-ocular elevada, edema macular cistóide, sinéquias posteriores, e, encarceramento da LIO pela íris ${ }^{(1)}$, opacificação da $\mathrm{LIO}^{(14)}$.

Um detalhe de extrema relevância é o tamanho da capsulorrexe, que quando muito pequena aumenta a probabilidade de síndrome de retração capsular, ou fimose capsular, podendo levar a descentração e até mesmo ao seu deslocamento em relação ao plano iriano ${ }^{(2)}$.

Os autores evitaram o posicionamento horizontal, por acreditarem que esta posição aumenta a incidência de descentração da LIO, sobretudo no pós-operatório imediato, embora este fato não esteja relatado na literatura (ao menos com as lentes hidrofílicas expansíveis), mas foi observação de um dos autores, com experiência de mais de 4 mil lentes expansíveis hidrofílicas implantadas.

Uma observação interessante foi o baixo índice de opacificação da cápsula anterior (38 olhos ou 2,53\%), bem como índice de formação do anel de Soemmering (205 olhos ou $13,66 \%)$, quando comparados à literatura ${ }^{(10-16)}$. Acredita-se que a biocompatibilidade da LIO, principalmente por ser expandida em meio constituído também de humor aquoso, possa ser a explicação para tal fato. Ainda, estes números reforçam a teoria de que esta lente somente sofre descentrações maiores que $2 \mathrm{~mm}$, quando posicionada em um saco capsular com capsulotomia pequena ${ }^{(10)}$, e sujeita a fimose capsular.

$\mathrm{Na}$ análise estatística, os intervalos de confiança foram de 0,029 para OCP, 0,017 para opacificação de cápsula anterior e formação do anel de Soemmering. O que resulta em um teste de hipótese onde o valor $\mathrm{p}=0,669>0,05$ para OCP, concluindo-se que a incidência da OCP não dependeu, no estudo, do ano em que foi realizada a cirurgia. Quanto à fimose capsular, o valor de $\mathrm{p}=0,001<0,05$, então, a incidência de fimose nos olhos operados depende do ano em que foi realizada a cirurgia. $\mathrm{Ou}$ seja, fornece o suporte à teoria que capsulorrexe pequena aumenta a incidência de fimose capsular ${ }^{(10)}$.

As lentes hidrofílicas possuem uma excelente biocompatibilidade se comparada com as lentes de silicone e PMMA, tendo uma biocompatibilidade semelhante às lentes hidrofóbicas $^{(11,15,17)}$.

A biocompatibilidade das lentes acrílicas hidrofílicas, ficou bem demonstrada, em estudo comparativo entre lentes hidrofílicas, hidrofóbicas e de silicone, no qual encontra que as hidrofílicas apresentam melhor biocompatibilidade uveal e pior biocompatibilidade capsular em pacientes com uveítes ${ }^{(5)}$. Neste estudo, no entanto, as lentes hidrofílicas utilizadas eram lentes previamente expandidas, ao contrário das lentes Acqua $^{\circledR}$. Acredita-se, pelos números encontrados neste trabalho, que a expansão da lente dentro do saco capsular com absorção de humor aquoso aumenta a biocompatibilidade e reduz a incidência de OCP inclusive quando em olhos portadores de uveítes crônicas ${ }^{(18)}$. 
A incidência de OCP em pacientes com lentes de silicone e pacientes com lentes acrílicas, apresentou índices estatisticamente semelhantes para os dois grupos ${ }^{(14,19)}$, embora discretamente maior nos pacientes com LIO de silicone ${ }^{(19)}$.

Em outros trabalhos ${ }^{(11,18,20-21)}$ encontram-se dados semelhantes, relacionando também a incidência menor de OCP nas lentes acrílicas tanto hidrofílicas quanto hidrofóbicas ao desenho de "square edge", ou seja, bordas quadradas, ao nível da óptica da LIO, criando uma barreira física à migração celular.

\section{CONCLUSÃO}

Acredita-se que este estudo demonstra a eficácia das LIO acrílicas hidrofílicas expansíveis, quanto à opacificação de cápsula posterior, apresentando uma incidência de 4,6\%. Recomenda-se a utilização de capsulorrexe superior a $6 \mathrm{~mm}$ de diâmetro.

Quanto ao fato de não ter sido observado nenhum caso de opacificação da LIO, atribui-se à não utilização de qualquer corante para a realização da capsulorrexe. Não se observou qualquer impregnação de pigmento nas LIO de nenhum dos pacientes acompanhados.

Devido ao baixo custo da LIO hidrofílica expansível, quando comparado à LIO hidrofóbica, e a sua pequena curva de aprendizagem para inserção, possibilitando que cirurgiões pouco experientes possam implantá-las com sucesso pósoperatório, e considerando que em países onde boa parte da população é excluída das modernas técnicas da cirurgia da catarata, esta lente vem preencher uma importante lacuna, apresentando excelente razão custo/benefício.

\section{ABSTRACT}

Purpose: To evaluate prospectively the results of expandable acrylic intraocular lenses in patients submitted to phacoemulsification as regard posterior capsule opacification. Methods: The study group consisted of 830 patients, 1,200 eyes that underwent phacoemulsification from 1998 to 2002, by the same surgeon, using the same technique but with different diameters of capsulorrhexis, with expandable hydrophilic acrylic intraocular lens implantation. The mean follow-up was 2.4 years, ranging from 6 months to 4 years. Results: The total number of posterior capsule opacification was 54 cases. Conclusions: The incidence of posterior capsule opacification was $4.6 \%$ in these patients.

Keywords: Lens implantation, intraocular; Contact lenses, hydrophilic; Lens capsule, crystalline; Visual acuity; Phacoemulsification; Capsulorrhexis

\section{REFERÊNCIAS}

1. Crema AS. A máquina e o instrumental. In: Centurion V. Faco total. Rio de Janeiro: Cultura médica; 2000. p.8-12.

2. Boyce JF, Bhermi GS, Spalton DJ, El-Osta AR. Mathematical modeling of the forces between an intraocular lens and the capsule. J Cataract Refract Surg. 2002;28(10):1853-9

3. Cabezas-Leon M, Gracia-San Roman J, Garcia-Caballero J, Morente-Matas P. [Quality of life following cataract surgery] Arch Soc Esp Oftalmol. 2005;80(8): 449-56. Spanish

4. Blumenthal M. Expandable acrylic Acqua IOL facilitates implant procedure. Ocul Surg. News Europe/Asia Pacific Edition, 2001;7:1.

5. Abela-Formanek C, Amon M, Schauersberger J, Kruger A, Nepp J, Schild G. Results of hydrophilic acrylic, hydrophobic acrylic, and silicone intraocular lenses in uveitic eyes with cataract: comparison to a control group. J Cataract Refract Surg. 2002;28(7):1141-52. Comment in: J Cataract Refract Surg. 2003; 29(8): 1470 .

6. Camparini M, Macaluso C, Reggiani L, Maraini G. Retroillumination versus reflected-light images in the photographic assessment of posterior capsule opacification. Invest Ophthalmol Vis Sci. 2000;41(10):3074-9.

7. Bussab WO, Morettin PA. Estatística básica. 4a ed. São Paulo: Atual; 1987.

8. Gamerman D, Migon HS. Inferência estatística: uma abordagem integrada. Textos de métodos matemáticos. Rio de Janeiro: Editora do Instituo de Matemática da Universidade Federal do Rio de Janeiro; 1993.

9. Pinheiro JID. Análise estatística de dados. Rio de Janeiro: Editora do Instituo de Matemática da Universidade Federal do Rio de Janeiro; 1988.

10. Hollick EJ, Spalton DJ, Ursell PG, Pande MV, Barman SA, Boyce JF, et al. The effect of polymethylmethacrylate, silicone, and polyacrylic intraocular lenses on posterior capsular opacification 3 years after cataract surgery. Ophthalmology. 1999;106(1):49-54; discussion 54-5.

11. Vargas LG, Peng Q, Apple DJ, Escobar-Gomez M, Pandey SK, Arthur SN, et al. Evaluation of 3 modern single-piece foldable intraocular lenses: clinicopathological study of posterior capsule opacification in a rabbit model. J Cataract Refract Surg. 2002;28(7):1241-50.

12. Findl O, Menapace R, Sacu S, Buehl W, Rainer G. Effect of optic material on posterior capsule opacification in intraocular lenses with sharp-edge optics: randomized clinical trial. Ophthalmology. 2005;112(1):67-72.

13. Zemaitiene R, Jasinskas V, Barzdziukas V, Auffarth GU. Prevention of posterior capsule opacification using different intraocular lenses (results of one-year clinical study). Medicina (Kaunas). 2004;40(8):721-30.

14. Tappin MJ, Larkin DF. Factors leading to lens implant decentration and exchange. Eye. 2000;14 Pt 5:773-6.

15. Moosavi A, Fox P, Harrison M, Phillips GJ, Lloyd AW. Opacification of SC60B-OUV lens implant following routine phacoemulsification surgery: case report and EM study. Br J Ophthalmol. 2003;87(6):800-1.

16. Heyrman TP, McDermott ML, Ubels JL, Edelhauser HF. Drug uptake and release by a hydrogel intraocular lens and the human crystalline lens. J Cataract Refract Surg. 1989;15(2):169-75.

17. Pohjalainen T, Vesti E, Uusitalo RJ, Laatikainen L. Posterior capsular opacification in pseudophakic eyes with a silicone or acrylic intraocular lens. Eur J Ophthalmol. 2002;12(3):212-8.

18. Yong VK, Netto PA, Heng WJ, Yap EY, Lee HM, Ng MM, et al. Visually significant opacification of hydrophilic acrylic intraocular lenses-a clinico-pathological analysis. Eur J Ophthalmol. 2003;13(2):147-50.

19. Schmidbauer JM, Vargas LG, Apple DJ, Auffarth GU, Peng Q, Arthur SN, et al. [Millenniums update on posterior capsule opacification (PCO) scores, centration, biocompatibility and fixation of foldable intraocular lenses (IOL) - an analysis of 1,221 pseudophakic post mortem globes] Klin Monatsbl Augenheilkd. 2001;218(10):649-57. German.

20. Dorey MW, Brownstein S, Hill VE, Mathew B, Botton G, Kertes PJ, et al. Proposed pathogenesis for the delayed postoperative opacification of the hydroview hydrogel intraocular lens. Am J Ophthalmol. 2003;135(5):591-8.

21. Serpa Junior E, Wishart PK. Comparison of PMMA, foldable silicone and foldable acrylic hydrophobic intraocular lenses in combined phacoemulsification and trabeculectomy. Arq Bras Oftalmol. 2005;68(1):29-35. 